\title{
Simulação Médica no Ensino Universitário de Pediatria
}

\section{Medical Simulation in the University Teaching of Pediatrics}

Luísa Maria de Morais Macieiral Maria Del Carmen Bento TeixeiraI Jorge Manuel Andrade Saraiva ${ }^{I}$

\section{PALAVRAS-CHAVE}

- Modelos Anatômicos.

- Materiais de Ensino.

- Pediatria.

- Manequins.

- Educação Médica.

Recebido em: 25/11/2016

Aprovado em: 05/12/2016

\begin{abstract}
RESUMO
Objetivos: Demonstrar as vantagens do uso de manequins simuladores no ensino prático médico, em particular pediátrico, e estudar os diferentes tipos de manequins/modelos existentes, especificidades e utilização própria de cada um deles. Métodos: A formação médica baseada na transmissão teórica de conhecimentos e com aprendizagem clínica praticada em doentes reais não é viável atualmente, pois depende da oportunidade e disponibilidade dos doentes em determinado momento. Hoje em dia, a Medicina recorre a modelos de simulação médica, permitindo o desenvolvimento da proficiência necessária à execução de determinado procedimento. A simulação médica proporciona diversas vantagens: não requer doentes reais e disponíveis, possibilita repetição da técnica com correção de erros, aumenta o nível de confiança do aluno em suas capacidades e não oferece risco ao doente. Desde 2009, o ensino de Pediatria no Estágio de Saúde Infantil dos alunos do sexto ano do Mestrado Integrado em Medicina na Faculdade de Medicina de Coimbra contempla aulas práticas com modelos, onde os alunos treinam técnicas como: intubação pediátrica, manobra de Ortolani, punção venosa em membros superiores, punção lombar, intubação orotraqueal, auscultação do murmúrio vesicular, punção venosa, avaliação da fontanela normotensa e da hipertensão da fontanela. Esta componente prática de ensino estimula e agrada aos alunos, que todos os anos a elegem como uma das preferidas do Estágio de Saúde Infantil no âmbito do Mestrado Integrado. Resultados: Aquisição de conhecimentos e prática de técnicas médicas, invasivas e não invasivas, por parte dos alunos, recorrendo a modelos de simulação médica pediátricos, nomeadamente: intubação pediátrica, manobra de Ortolani, punção venosa em membros superiores, punção lombar, intubação orotraqueal, auscultação do murmúrio vesicular, punção venosa, avaliação da fontanela normotensa e da hipertensão da fontanela. Feita a avaliação destas mesmas aulas pelos alunos por meio de questionário de preferências, ficou claro que esta é uma das aulas preferidas dos alunos do sexto ano de Medicina em Estágio de Saúde Infantil do Mestrado Integrado em Medicina. Conclusões: Foi possível aos alunos desenvolver competências na realização de procedimentos médicos, com uma avaliação muito positiva pelos estudantes do Estágio em Saúde Infantil do Mestrado Integrado em Medicina.
\end{abstract}




\section{KEYWORDS}

- Models, Anatomic.

- Teaching Materials.

- Pediatrics

- Mannequins

- Medical Education.
ABSTRACT

Objectives: To demonstrate the advantages of using simulator mannequins in practical medical education and particularly in pediatrics, studying the different kinds of existing mannequins/models, their specific characteristics and uses. Methods: Medical training based on the theoretical transmission of knowledge and clinical learning practiced on real patients is currently unviable because it is dependent on the timing and availability of patients at any given moment. Nowadays, medicine uses medical simulation models, thus facilitating the development of proficiency in skills required for the execution of particular procedures. Medical simulation provides several advantages: it does not require real and available patients, techniques may be repeated in order to address errors, students' confidence in their abilities is promoted, and there is an absence of risk for the patient. Since 2009, the child health internship undertaken as part of the pediatric education section of the Integrated Master's degree at the University of Coimbra Faculty of Medicine by sixth-year students has included practical classes with models where students practice techniques such as pediatric intubation, the Ortolani maneuver, venipuncture in upper limbs, lumbar puncture, endotracheal intubation, breath sounds auscultation, venipuncture, evaluation of normotensive fontanelle, and hypertension fontanelle. This practical component stimulates and appeals to students, who repeatedly select these classes as one of their favorites on the child health internship completed as part of the Integrated Master's degree in Medicine. Results: Students used medical simulation models to acquire knowledge and practice medical, invasive and non-invasive techniques such as: pediatric intubation, the Ortolani maneuver, venipuncture in the upper limbs, lumbar puncture, endotracheal intubation, breath sounds auscultation, venipuncture, evaluation of normotensive fontanelle, and hypertension fontanelle. Upon the students' assessment by means of a preference questionnaire, it became clear that these classes were some of those preferred by sixth-year students completing the child health internship as part of their Integrated Master's degree. Conclusions: Students completing child health internships as part of the Integrated Master's degree in medicine were able to develop skills in performing medical procedures, with very positive assessments given by these students on the experience.

\section{INTRODUÇÃO}

Criada em 1290 pelo rei D. Dinis, a Universidade de Coimbra é a mais antiga de Portugal e uma das mais antigas do mundo, presumindo-se que o ensino de Medicina em Coimbra seja até anterior a este período (1131).

A Faculdade de Medicina da Universidade de Coimbra (FMUC) é, neste momento, frequentada por 1.980 alunos, sendo a média de ingresso uma das mais altas do país $(\geq 18$ valores, numa escala de 0 a 20). A FMUC alberga estudantes de vários pontos do mundo, sendo de cerca de 150 o número de estudantes em mobilidade internacional incoming por ano, oriundos de vários países, nomeadamente o Brasil.

O curso de Medicina-Mestrado Integrado da FMUC é composto por ciclo básico e segundo ciclo, num total de seis anos. O sexto ano Pós-Bolonha (desde 2000) é constituído por estágios práticos programados e orientados nas várias áreas (Cirurgia, Medicina Interna, Saúde Materna, Saúde Mental, Saúde Pública, Oncologia, Medicina Geral e Familiar, e Saúde
Infantil). Durante estes estágios, os alunos tomam contato com as realidades médicas e desafios próprios de cada setor.

A fim de garantir uma boa aprendizagem dos conhecimentos nestas áreas, a parte prática é essencial, contudo pode ser limitante se usarmos doentes reais. A formação médica baseada na transmissão teórica de conhecimentos e com uma aprendizagem clínica exclusivamente praticada em doentes reais depende da oportunidade e disponibilidade de doentes em determinado momento, o que não é viável nos dias de hoje. A constante evolução na prática clínica, assim como imperativos éticos e a necessidade de rentabilização de recursos humanos e hospitalares inviabilizam que o ensino e treino de determinados procedimentos médicos, em particular os invasivos, sejam efetuados em doentes reais. Daí a necessidade de novos métodos de transmissão de conhecimentos e de avaliação de atos práticos clínicos.

A simulação médica proporciona diversas vantagens como método de ensino e de avaliação: não necessita de doen- 
tes reais e disponíveis, possibilita a repetição da técnica com correção de erros, aumenta o nível de confiança do aluno em suas capacidades e não apresenta risco para o doente, permitindo que o aluno desenvolva a proficiência necessária à execução de determinado procedimento médico ${ }^{1}$. Para isto, utilizam-se modelos e manequins que simulam o doente real. Esta técnica de ensino-aprendizagem serve para a transmissão e aquisição de aspectos cognitivos (conhecimentos fundamentais de base) e psicomotores (gestos, técnicas e procedimentos para determinado protocolo clínico), permitindo aos alunos o contato com cenários e procedimentos clínicos progressivamente mais complexos, com possibilidade de feedback sobre a própria prática, num contexto reprodutível e estandardiza$\mathrm{do}^{1,2}$. Tudo isto sem pôr em causa a segurança dos doentes, fato particularmente importante quando se trata de doentes pediátricos.

Historicamente, apenas nas últimas duas décadas é que o ensino por simulação foi adaptado à área da Medicina, como resultado do impulso de diversos estudos de validação científica deste método e da relação custo-efetividade na prática dos futuros profissionais nesta área, tal como demonstrado por Bosse ${ }^{3}$.

Por meio do método de aprendizagem por simulação, o discente pode cometer erros e aprender a corrigi-los continuamente, adquirindo a competência necessária para executar determinada técnica clínica de forma correta e garantir a segurança dos futuros doentes ${ }^{3-6}$.

Este método de ensino implica o uso de equipamentos (manequins e demais materiais) necessários para criar um cenário o mais real possível. Tal como Amaral salienta, existem diversos tipos de manequins: além dos tradicionais manequins de corpo inteiro e de modelos de partes anatômicas (modelos low-tech) - ambos disponíveis para diversas faixas etárias -, também existem manequins de alta fidelidade (modelos high-tech), que consistem em manequins robotizados, nos quais está incorporado um software acoplado a um computador, permitindo ao manequim ter as reações naturais que um doente real teria (tosse, choro, dor, reações inflamatórias, etc.) ${ }^{2}$.

O uso de manequins de simulação permite o ensino e avaliação de diversas técnicas médicas, como medição da pressão arterial, oftalmoscopia, otoscopia, auscultação cardiopulmonar, intubações, aplicação de venóclise, mudança posicional de doentes e seu transporte, punção lombar, cateterismo da artéria ou veia umbilical nos recém-nascidos, manobra de Ortolani, prática com desfibrilador, entre outras.

Para que o seu uso seja possível, há que ter em atenção diversos aspectos logísticos, adaptáveis à realidade de cada instituição:
- disponibilidade dos modelos necessários ao tipo de técnica a executar;

- demais materiais necessários à execução do protocolo (seringas, agulhas, batas, máscaras, luvas, cateteres, sondas de aspiração, tubos, compressas, etc.);

- explicação detalhada do procedimento, acompanhada de exemplificação do professor e de protocolo escrito;

- formação de pequenos grupos de alunos para treino da técnica;

- feedback, correção de erros, esclarecimento de dúvidas;

- execução repetida da técnica até aquisição da competência pelos alunos ${ }^{7}$.

Um aspecto importante a ter em conta quando se usa o método de ensino por meio de manequins e modelos é o seu custo de aquisição e de manutenção. Em média, um manequim convencional de idade pediátrica pode custar de 800 a $4.000 €$, e um manequim de alta fidelidade (robotizado e com software), 80.000 a $250.000 €$. Além do custo da sua aquisição, há que ter em conta também os custos de manutenção. Assim, há que rentabilizar os recursos existentes ${ }^{2}$.

Desde 2009, o ensino de Pediatria no estágio de Saúde Infantil dos alunos do sexto ano do Mestrado Integrado em Medicina na Faculdade de Medicina da Universidade de Coimbra contempla aulas práticas nas quais os alunos podem executar e treinar manobras invasivas e não invasivas em modelos em escala real, após introdução teórica adequada com explicação das diversas técnicas e realização das mesmas pelo docente responsável por essas aulas.

Os objetivos deste trabalho são demonstrar as vantagens do uso de manequins simuladores no ensino prático médico, em particular pediátrico, além de estudar os diferentes tipos de manequins/modelos existentes e as especificidades e utilização própria de cada um deles.

\section{MATERIAL E MÉTODOS}

Nas aulas práticas do Estágio de Saúde Infantil do Mestrado Integrado em Medicina na Faculdade de Medicina da Universidade de Coimbra, os alunos aprendem diversas técnicas usando manequins pediátricos inteiros e parciais:

- manobra de Ortolani (Figura 1);

- membros superiores para prática de punção venosa (Figura 2);

- punção lombar (Figura 3);

- intubação pediátrica (Figura 4);

- intubação orotraqueal, auscultação do murmúrio vesicular, punção venosa, avaliação da fontanela normotensa e da hipertensão da fontanela (Figura 5). 


\section{Figura 1}

Modelo onde se executa a manobra de Ortolani no despiste de luxação congênita da anca (este modelo apresenta luxação congênita da anca bilateral)

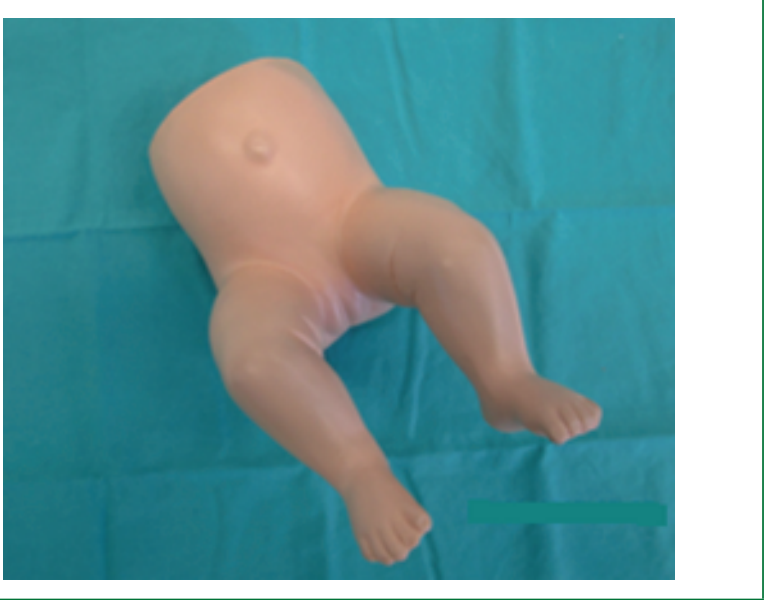

Além das vantagens já abordadas do uso destes manequins, estas aulas despertam o interesse dos alunos dada a componente realista e a forte aproximação da prática real da atividade médica pediátrica do dia a dia. Isto faz com que esta seja uma das aulas práticas consecutivamente preferidas pelos alunos no decorrer do Estágio em Saúde Infantil ao longo dos seus anos de existência, como demonstra a Figura 6. Para isto, foi usado um questionário contendo todas as valências lecionadas durante o estágio em Saúde Infantil, no qual os alunos atribuíam uma pontuação a cada uma delas, sendo: 1 - muito fraco; 2 - fraco; 3 - razoável; 4 - bom; 5 - muito bom.

Figura 2

Membros superiores para prática de punção venosa

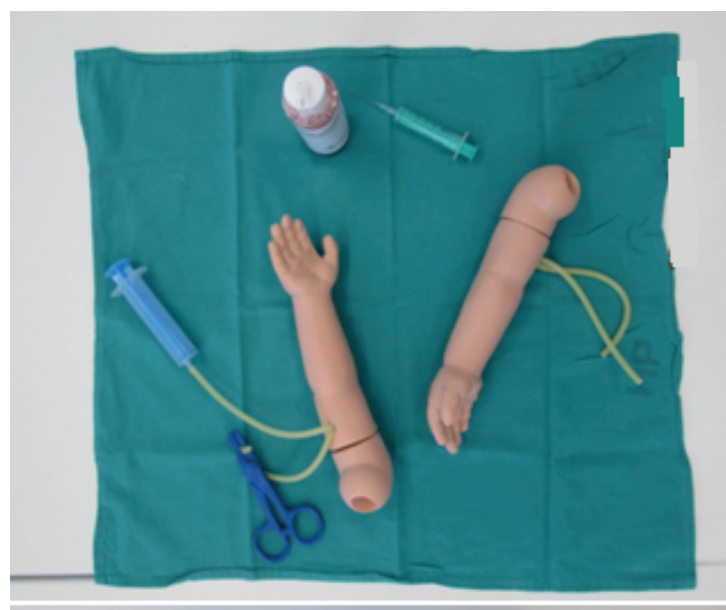

Figura 3

Modelo onde se realiza a punção lombar

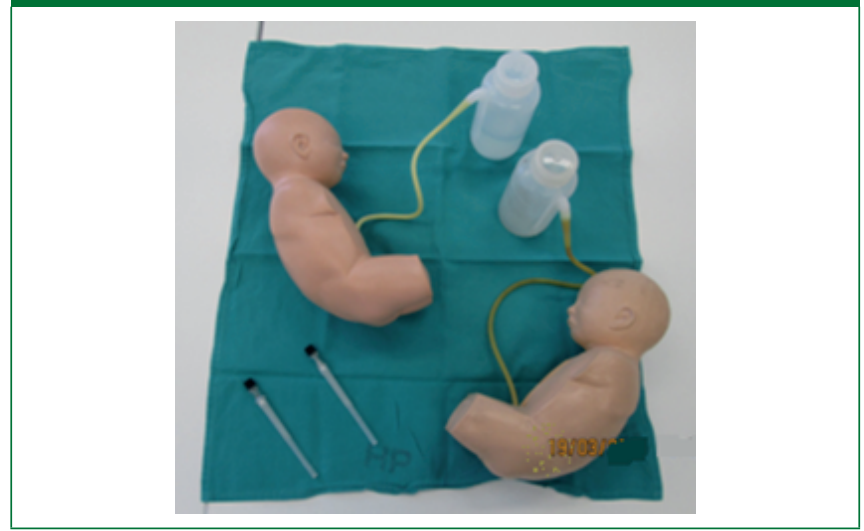

Figura 4

Modelo para treino de intubação pediátrica

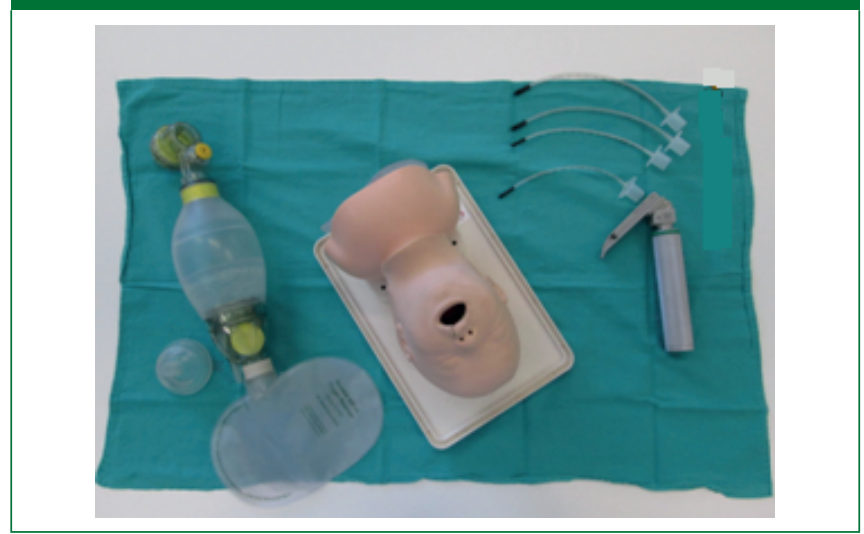

Figura 5

Modelo multifunções: intubação orotraqueal com auscultação do murmúrio vesicular, punção venosa e avaliação das características de uma fontanela normotensa e de uma hipertensão da fontanela

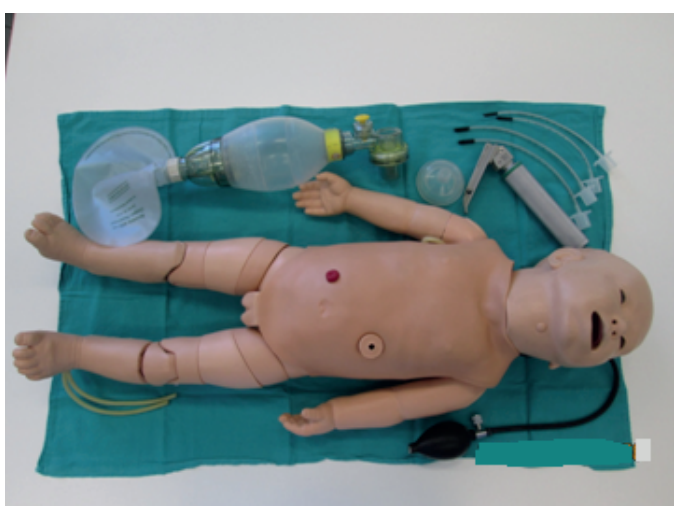


Figura 6

Gráfico demonstrativo das preferências dos alunos

relativamente às aulas práticas no Estágio em

Saúde Infantil no ano letivo de 2013-2014. Médias

das avaliações das atividades de aprendizagem.

As avaliações dos alunos foram feitas segundo

a seguinte codificação: 1 - muito fraco; 2 -

fraco; 3 - razoável; 4 - bom; 5 - muito bom

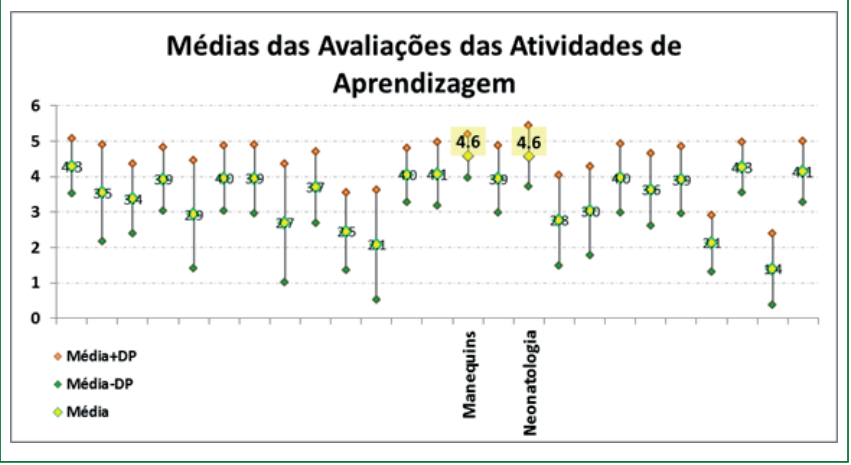

Como é visível pela análise do gráfico, as aulas de Pediatria com uso de manequins são a segunda opção mais classificada com o nível 5. Tal preferência mantém-se ao longo dos anos, alternando entre a segunda e a primeira posição.

\section{RESULTADOS E DISCUSSÃO}

Graças ao uso destes modelos, os alunos do sexto ano do Mestrado Integrado em Medicina aprenderam a realizar corretamente as seguintes técnicas médicas:

- auscultação do murmúrio vesicular (Figura 5);

- avaliação das características da fontanela normotensa (Figura 5);

- avaliação da hipertensão da fontanela (Figura 5);

- manobra de Ortolani (Figura 1);

- punção venosa (Figura 2);

- punção lombar (Figura 3);

- intubação pediátrica (Figura 4);

- intubação orotraqueal (Figura 4).

Esta técnica de ensino por simulação com manequins em escala real permitiu aos alunos, além da aquisição das competências acima referidas, realizar estes procedimentos às vezes necessários, corrigindo seus erros até se sentirem à vontade e capazes de fazê-los corretamente sozinhos. Também é de referir que este método de ensino lhes permitiu, à medida que foram aprendendo e executando técnicas cada vez mais complexas, constatar suas principais dificuldades e aprender a contorná-las. Isso lhes dá a competência necessária para executá-las e confiança para quando chegar a hora de realizar estes procedimentos em doentes reais. Tratando-se de doentes pediátricos, a confiança que o médico transmite à criança e aos pais é de extrema importância e fulcral para o processo de tratamento/cura da criança.

Independentemente do tipo de modelo/manequim usado, esta técnica de ensino apresenta várias vantagens:

- possibilidade de repetição do procedimento;

- possibilidade de correção de erros;

- o aluno toma conhecimento das principais dificuldades de cada técnica aprendida;

- não põe em causa a saúde do doente;

- o sujeito (modelo) está sempre disponível para praticar (ao contrário dos doentes reais).

A utilidade e a importância deste tipo de ensino com modelos e manequins para estas aulas práticas são notórias, em todos os seus fatores. De tal forma, que até os alunos que frequentam estas aulas práticas o demonstram, assinalando-a como uma das preferências de topo entre as aulas deste estágio. Como se pode ver na Figura 6, no ano transato esta aula foi a segunda eleita pelos alunos deste estágio na ordem de preferências entre as aulas práticas lecionadas no decorrer do Estágio em Saúde Infantil.

Daqui concluímos que o uso de modelos para as aulas práticas em Medicina é de vital importância, pois permite a aquisição das competências necessárias sem pôr em causa a saúde do doente, atribuindo aos futuros médicos a competência e a confiança necessárias para realizarem da melhor forma possível o seu trabalho no dia a dia.

\section{REFERÊNCIAS}

1. Lopreiato JO, Sawyer T. Simulation-Based Medical Education in Pediatrics. Academic Pediatrics. 2015;2:134-142.

2. Amaral JMV. Simulação e ensino-aprendizagem em Pediatria. 1 $1^{\text {a }}$ Parte: Tópicos essenciais. Acta Pediátrica. 2010,41:44-50.

3. Bosse HM, Mohr J, Buss B, Krautter M, Weyrich P, Herzog $\mathrm{W}$, et al. The benefict of repetitive skills training and frequency of expert feedback in the early acquisition of procedural skills. BMC Medical Education. 2015,15:286.

4. Dent J, Harden R. A Practical Guide for Medical Teachers. Edinburgo: Elsevier-Churchill Liningstone; 2005.

5. Henriksen K, Battles JB, Marks ES, Lewin DI. Advances in Patient Safety: From Research to Implementation. Volume 4: Programs, Tools and Products. Rockville (MD): Agency for Healthcare Research and Quality (US); 2005. 
6. Henrinksen K, Battles JB, Keyes MA, Grady ML. Advances in Patient Safety: New Directions and Alternative Approaches. Volume. 3: Performance and Tools. Rockville (MD): Agency for Healthcare Research and Quality (US); 2008.

7. Camilo H. A educação médica baseada na simulação e em simuladores. Essências Educare. 2008, 8. Portugal.

\section{CONTRIBUIÇÃO DOS AUTORES}

Luísa Macieira concebeu e desenhou o estudo, recolheu e analisou os dados, escreveu o primeiro rascunho do texto e fez posterior revisão. Aprovou a versão final do texto. Maria Del Carmen Bento concebeu e desenhou o estudo, analisou os dados e contribuiu para a escrita e revisão do texto. Aprovou a versão final do texto. Jorge Manuel Andrade Saraiva concebeu e desenhou o estudo e contribuiu para a escrita e revisão do texto. Aprovou a versão final do texto.

\section{CONFLITO DE INTERESSES}

Os autores declaram não haver conflito de interesses.

\section{ENDEREÇO PARA CORRESPONDÊNCIA}

Luísa Maria de Morais Macieira

Hospital Pediátrico Carmona da Mota - Centro Hospitalar e Universitário de Coimbra

Av. Afonso Romão, 3000-602 - Coimbra

E-mail: luisa_macieira@hotmail.com; carmenbento@sapo.pt; j.saraiva@chc.min-saude.pt 